\title{
Dynamic Effect of Cushion Part of Wheeled Vehicles on Their Steerability
}

\author{
B. Sokil ${ }^{1}$, O. Lyashuk ${ }^{2 *}$, M. Sokil ${ }^{3}$, P. Popovich ${ }^{4}$, Y. Vovk ${ }^{4}$ and O. Perenchuk ${ }^{2}$ \\ ${ }^{1}$ Department of Engineering Mechanics, Hetman Petro Sahaidachnyi National Army \\ Academy, 32 Heroes of Maidan street, 79012 Lviv, Ukraine, \\ ${ }^{2}$ Department of Automobile Transport, Ternopil Ivan Pul'uj National Technical \\ University, 56 Rus'ka street, 46001 Ternopil, Ukraine \\ Email: Oleg-lashyk@ rambler.ru \\ Phone: +380352519710; Fax: +380352254983 \\ ${ }^{3}$ Department of Transport Technologies, Lviv Polytechnic National University, \\ 12 Stepan Bandera street, 79000 Lviv, Ukraine \\ ${ }^{4}$ Department of Transport Technologies and Mechanics, \\ Ternopil Ivan Pul'uj National Technical University, 56 Rus'ka street, 46001 Ternopil, \\ Ukraine
}

\begin{abstract}
The effect of relative motion of cushion system on dynamic pressure force of steered wheeled vehicles with non-linear elastic characteristics of suspension was evaluated in the article under consideration. The problem is to find the dynamic force of system cushion-non-cushion part pressure on front wheel. The dependence for the limiting value of dynamic turning angle of steered wheels on longitudinal-and-angular oscillations amplitude and parameters describing elastic characteristics of elastic suspension was obtained. It was demonstrated that for more rigid suspensions the value of dynamic turning angle is smaller at small amplitudes of longitudinal-and-angular oscillations and bigger at big amplitudes of longitudinal-and-angular oscillations.
\end{abstract}

Keywords: Wheeled vehicles steerability; limiting value of steered wheels turning angle; longitudinal-and-angular oscillations; amplitude; frequency.

\section{INTRODUCTION}

One of the operating characteristics of wheeled vehicles (WV) is steerability $[1,2]$. It implies the ability of changing the traffic route of a car and keeping to the given direction due to the driver's action on the steering wheel, that finally results in an angle change between motion velocity vector of WV and steered wheels plane. Car steerability depends on both internal and external factors, which are to a certain degree interdependent.

Road covering characteristics determine the tire turning moment of resistance, to be more exact, its main component - the tire turning moment of resistance [1, 3-5], which depends on the coefficient of road-holding capacity of a tire, wheel load, its toe-in etc. The relative motion performance of CS is affected both by internal factors (WV geometry, mass distribution, elastic characteristics of CS and others) and external ones as well - its motion disturbance (road bumpiness). The latter depends on the coefficient of roadholding capacity of a tire, wheel load, its toe etc. If a vehicle is travelling on a bumpy road, then the dynamic wheel load is determined both by relative and transportation motion of cushioning system (CS) [6, 7]. A mathematical model of the vibrating system 
equivalent to the vehicle in the mode of emergency braking is developed and the solution is presented [8-10].

Rather sophisticated models for numerical simulation of vehicle dynamics on an uneven road are known [11-13]. The main goal of these models is the calculation of vertical vibrations in the context of comfort issues, damping technique and additional loading on the vehicle parts. The success of a dynamical model depends, first, on the quality of its validation which can be a rather difficult problem in the case of many degrees of freedom and unknown parameters. The concept of vibrational mechanics makes it possible to present the final result for averaged motion as a standard equation. This makes the further interpretation of the problem and its solution especially clear.

Thus, the matter of WV steerability should be considered comprehensively taking into account CS performance. Unfortunately, the matter of WV CS relative and translated performance effect on dynamic force value of steered wheels pressure, i.e. tire turning moment of resistance (WV steerability) has not been properly taken into consideration in the research dealing with this problem. Hence, the purpose of the paper is the estimation of effect of relative motion of the vehicle cushion system on dynamic pressure force of steered wheels with non-linear elastic characteristic of suspension by means of minimal model and vibrational mechanics prospects. It allows us not only to estimate the vehicle motion resistance analytically to describe certain quality features of the car behavior [14, 15]. The research results have great importance for the development of active/passive safety control systems and for ensuring driving safety.

\section{MATERIAL AND METHOD}

For a two-dimensional model of WV, shown on Figure 1 we have to find dynamic force of steered wheels pressure on bearing surface (road) taking into account main internal factors of non-cushion-cushion parts system. It is the dynamic force that plays the leading role in the tire turning moment of resistance finding. The work under discussion studies only longitudinal-angular oscillations effect on WV steerability, so we suppose:

a) suspension system is characterized by elastic forces and forces of resistance, described by dependencies $[6,7,16] \mathrm{F}_{\text {app. }}=\tilde{n}_{i} \Delta_{i}^{v+1}, \mathrm{R}_{\text {res. }}=\alpha_{i} \dot{\Delta}_{i}^{s+1}$, where $\tilde{n}_{i}, \alpha_{i}, s-$ steels, $\Delta_{i}$ and $\dot{\Delta}_{i}$ spring dampers deformation and its velocity accordingly ( $i=1$ for front suspension and ${ }^{3}=2-$ for back suspension);

b) maximum value of CS elastic force during CP longitudinal-angular oscillations is much bigger than maximum value of damper device resistance force;

c) $\mathrm{CP}$ center of gravity and car wheelbase ratio is determined by the parameters $\grave{a}$, $b, c$ (Figure 1).

d) $\mathrm{CP}$ in the process of motion produces some small oscillations around center of gravity $c(O)$ and its relative position is definitely determined by parameter $\varphi$ (relative turning angle around stated center and equilibrium position ratio);

e) elastic characteristics of wheels may be neglected or $\mathrm{F}_{i \text { pрp. }}=\tilde{n}_{i} \Delta_{i}^{v+1}$ may be considered as net force of dampers force of elasticity and wheels elastic force. 


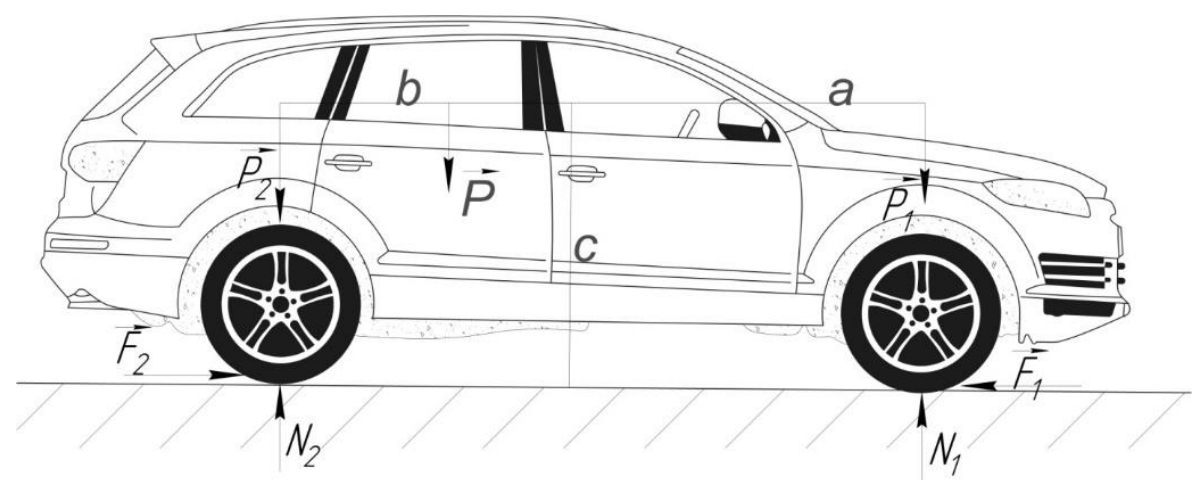

Figure 1. Calculation model and distribution of external forces acting on WV.

\section{SOLUTION PROCEDURE}

To answer the problem under discussion we use corollaries of d'Alambert principles for non-cushion-cushion part of mechanic system. Hence

$P_{1}+P_{2}+P-N_{1}-N_{2}=0$

$F_{2}-F_{1, f r}=0$

$\left(N_{2}-P_{2}\right)(a+b)-P a+M_{A}^{\circ}=0$,

where $P, P_{1}, P_{2}$ correspondingly $\mathrm{CP}$ weight, back and front axles, $F_{2}, F_{1, r .}$ driving and resistance forces, $N_{1}, N_{2}$ - normal constituents of road reactions, $M_{A}^{\Phi}-$ inertia forces moment of CP about a contact point (touch) steered wheel and road covering (p. A). System of algebraic Eq. (1) determine unknown reactions.

$$
\begin{aligned}
& N_{1}=P_{1}+P-\frac{P a-M_{A}^{\hat{o}}}{a+b}, \\
& N_{2}=P_{2}+\frac{P a-M_{A}^{\hat{o}}}{a+b}, \\
& F_{2}=F_{1 f r .} .
\end{aligned}
$$

The paper takes into consideration the case of WV motion on the road with constant speed (it follows from the last ratio of Eq. (2)). More complex case of accelerated $F_{2}>F_{1 f r}$ or decelerated $F_{2}<F_{1 f r}$ vehicle motion may be the subject-matter of further research.

The matters of road bumpiness influence on CP oscillations aren't covered in the article. They may be also the subject-matter of further research. In the paper under consideration we suppose that due to road bump CP got the initial motion disturbance (initial oscillations amplitude).

It seems that the problem has already solved but in first two ratios of dependencies Eq. (2) inertia forces moment in its relative motion about a p.A is still undetermined. Its determination is much more difficult task, as we consider WV with non-linear force characteristic of CS. The basis for its determination can be a differential equation of small relative longitudinal-angular oscillations of $\mathrm{CP}$

$$
I_{o} \ddot{\varphi}=-a\left(F_{\text {lapp. }}+R_{\text {Ires. }}\right)-b\left(F_{2 a p p .}+R_{\text {lres. }}\right) \text {, }
$$


where $I_{o}$ inertia moment of $\mathrm{CP}$ against horizontal axis which is crossing the center of gravity and is perpendicular to velocity vector of WV transportation motion, i.e., $I_{o}=P /(3 g)\left(a^{2}+b^{2}+c^{2} / 4\right), \varphi(t)-$ deviation at optional time against equilibrium position of CP. As it was mentioned above, the aim of the paper is to study longitudinal-angular oscillations on WV steerability. In the case with mentioned above oscillations of $\mathrm{CP}$ with accuracy to values of second order the elastic forces can be written as:

$$
F_{\text {lapp. }}=c_{1}\left(\varphi a-\Delta_{s t}\right)^{v+1}, F_{2 a p p .}=c_{2}\left(\varphi b+\Delta_{\text {st. }}\right)^{v+1} \text {. }
$$

where $\Delta_{\text {st. }}-$ static deformation of elastic elements. It allows differential Eq. (3) to be written as

$$
I_{o} \ddot{\varphi}+\left(c_{1} a^{v+2}+c_{2} b^{v+2}\right) \varphi^{v+1}=(v+1) \Delta_{s t}\left(c_{1} a^{v+1}-c_{2} b^{v+1}\right) \varphi^{v}-\left[\alpha_{1} a^{s+2}+\alpha_{2} b^{s+2}\right] \dot{\varphi}^{s+1} .
$$

It's not possible to construct the exact solution. But the restrictions imposed above concerning the system allow to use for it general ideas of disturbance methods $[17,18]$. Efficiency of their use greatly depends on the possibility of solution construction of equation undisturbed analogue (5), i.e. the equation

$$
I_{o} \ddot{\varphi}_{0}+\left(c_{1} a^{v+2}+c_{2} b^{v+2}\right) \varphi_{0}^{v+1}=0
$$

Eq. (5) and Eq. (6) will describe oscillation process of CP, if parameter $v+1$ in them is determined by ratio $v+1=(2 m+1) /(2 n+1) .(m, n=0,1,2, .$.$) . Besides, periodic$ solution of equation (6) in this case is expressed in terms of periodic Ateb-functions [1921].

$$
\varphi_{0}(t)=a_{\varphi} c a\left(v+1,1, \omega\left(a_{\varphi}\right) t+\theta\right)
$$

where $a_{\varphi}, \omega\left(a_{\varphi}\right)=\sqrt{\left(c_{1} a^{v+2}+c_{2} b^{v+2}\right)(v+2) /\left(2 I_{0}\right)} a_{\varphi}^{\frac{v}{2}}-$ amplitude and frequency of CP free longitudinal-angular oscillations, $\omega\left(a_{\varphi}\right) t+\theta-$ their phase. Free oscillations frequency may be replaced by more comfortable dependence for these reasons: if "rigidity" parameters of spring dampers $\mathrm{WV} \mathrm{CP} c_{1}, c_{2}$ are related by ratio $c_{2}=\kappa c_{1}(\kappa-$ known constant), then it will be more reasonable to use the concept of static deformation of elastic bumpers $-\Delta_{\mathrm{st}}$. In this case $c_{1}=P /\left((1+\kappa) \Delta_{\mathrm{st}}^{v+1}\right)$, and frequency of free oscillations takes the following value

$$
\omega\left(a_{\varphi}\right)=\sqrt{P\left(a^{v+2}+\kappa b^{v+2}\right)(v+2) /\left(2(1+\kappa) I_{0} \Delta_{s .}^{v+1}\right)} a_{\varphi}^{\frac{v}{2}}
$$

The given ratios and plots constructed on their basis show one of the essential oscillations difference of WV CP with CS non-linear characteristic comparing to its linear analogue - free oscillations frequency of WV CP depends on amplitude, besides, for CS with progressive characteristic of spring dampers the bigger longitudinal-angular oscillations amplitude the higher own frequency value, for regressive one - vice versa: the bigger amplitude, the lower frequency. In Figure 2 amplitude-frequency ratio is given 
for different values of CS force characteristics with $P=10^{5} \mathrm{~N}, P_{1}=10^{4} \mathrm{~N}, a=c=1 \mathrm{~m}$, $b=1,1 m, \kappa=1,2$.

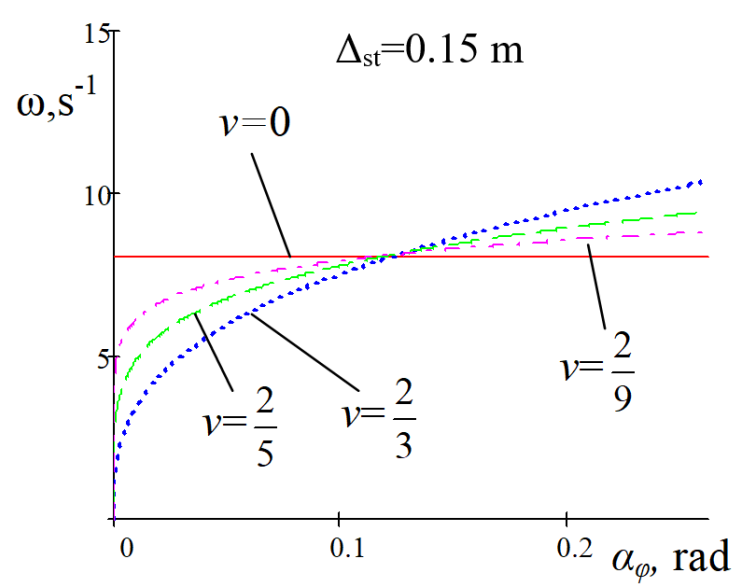

(a)

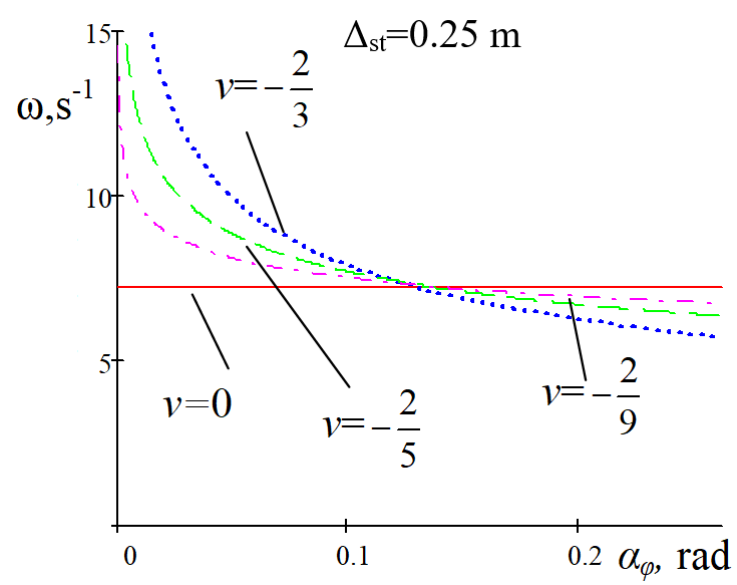

(c)

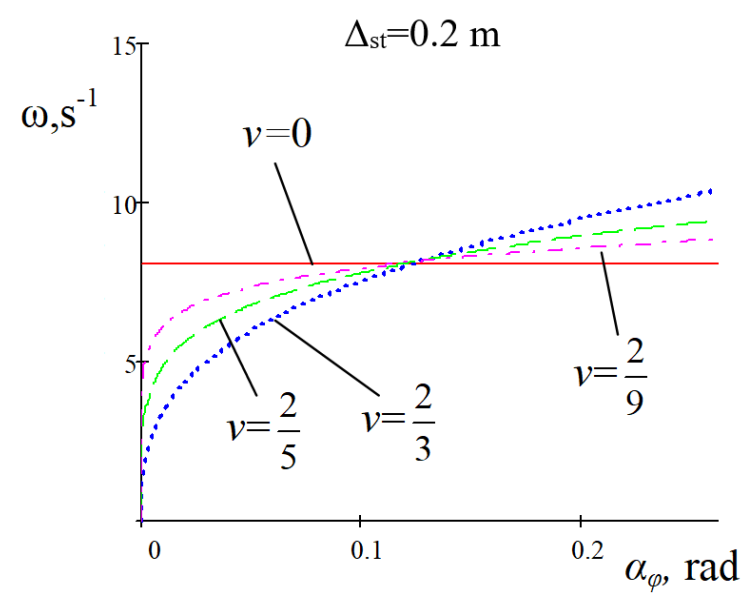

(b)

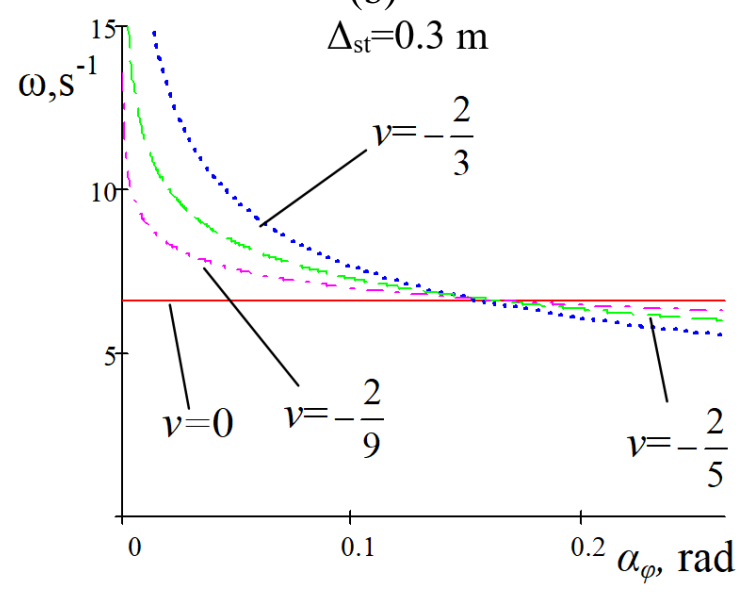

(d)

Figure 2. Frequency-amplitude ratio of $\mathrm{CP}$ own longitudinal-angular oscillations at different CS force characteristics values with progressive (a), (b) and regressive laws of elastic force change (c), (d).

For undisturbed Eq. (6) parameters $a_{\varphi}, \theta$ are constant, as corresponding system is conservative. As for resistance forces and other non-linear forces effect, which analytic approximation is presented in the right part of Eq. (5), it results in oscillations amplitude damping, i.e. damping oscillations frequency change. The easiest way to determine the above-mentioned forces impact is using the main idea of Van-der-Pol method [18] concerning the given type of system. According to his main idea the first asymptotic approximation describing CP longitudinal-angular oscillations taking into account resistance forces, is expressed as

$\varphi_{0}(t)=a_{\varphi}(t) c a\left(v+1,1, \omega\left(a_{\varphi}\right) t+\theta(t)\right)$

where $a_{\varphi}(t)$ and $\theta(t)$ - unknown variables in function time, which laws of variation are determined in right part of the Eq. (5). To determine them by time differentiation the 
Eq. (9) we have $\frac{d \varphi_{0}}{d t}=-2 /(v+2) \omega\left(a_{\varphi}\right) s a\left(1, v+1, \omega\left(a_{\varphi}\right) t+\theta\right)$. Similarly, we find the first-order derivative by disturbed motion time:

$$
\frac{d \varphi}{d t}=-\frac{2}{v+2} \omega\left(a_{\varphi}\right) s a\left(1, v+1, \omega\left(a_{\varphi}\right) t+\theta\right)+\frac{d a_{\varphi}}{d t} c a(v+1,1, \omega t+\theta)-\frac{2}{v+2} \frac{d \theta}{d t} \omega(a) s a(1, v+1, \omega t+\theta) .
$$

By next differentiation of the given dependence, taking into account $\frac{d a_{\varphi}}{d t} c a(v+1,1, \omega t+\theta)=\frac{2}{v+2} \frac{d \theta}{d t} \omega(a) s a(1, v+1, \omega t+\theta)=0$, we have;

$$
\begin{aligned}
& \frac{d^{2} \varphi(t)}{d t^{2}}=-\frac{2 \omega^{2}\left(a_{\varphi}(t)\right)}{v+2} a_{\varphi} c a^{v+1}\left(v+1,1, \omega\left(a_{\varphi}(t)\right) t+\theta(t)\right)-\frac{2}{v+2} \times \\
& \times \frac{d a_{\varphi}}{d t}\left[\omega\left(a_{\varphi}(t)\right)+a \frac{d \omega\left(a_{\varphi}\right)}{d a_{\varphi}}\right] s a\left(1, v+1, \omega\left(a_{\varphi}(t)\right) t+\theta(t)\right)-\frac{2 \omega\left(a_{\varphi}(t)\right)}{v+2} \frac{d \theta}{d t} a c a\left(v+1,1, \omega\left(a_{\varphi}(t)\right) t+\theta(t)\right) .
\end{aligned}
$$

It's easy to make sure, that ratio $\omega\left(a_{\varphi}\right)+a_{\varphi} \frac{d \omega\left(a_{\varphi}\right)}{d a_{\varphi}}$ can be replaced by a simpler one, namely $(v+2) \omega\left(a_{\varphi}\right) / 2$. It allows us to obtain from the differential Eq. (5) the ratio describing CP longitudinal-angular oscillations - usual first-order differential equation, linking amplitude and phase of longitudinal-angular oscillations of disturbed motion

$$
\frac{d a_{\varphi}}{d t} s a\left(1, v+1, \omega\left(a_{\varphi}\right) t+\theta\right)+\frac{d \varphi}{d t} \frac{2}{v+2} a_{\varphi} c a^{v+1}\left(v+1,1, \omega\left(a_{\varphi}(t)\right) t+\theta(t)\right)=\bar{f}\left(a_{\varphi}, \bar{\theta}\right),
$$

where $\bar{\theta}=\omega\left(a_{\varphi}\right) t+\theta, \bar{f}\left(a_{\varphi}, \bar{\theta}\right)$. corresponds the right part value of Eq. (5) under condition that function $\varphi(t)$ and its first derivative are determined according to undisturbed case. In total, all given above allow us to find ratios describing laws of variations of $\mathrm{CP}$ oscillations amplitude and frequency as:

$$
\begin{aligned}
& \frac{d a_{\varphi}}{d t}=-\frac{1}{\omega\left(a_{\varphi}\right)} s a(1, v+1, \bar{\theta}) \bar{f}\left(a_{\varphi}, \dot{\bar{\theta}}\right) ; \\
& \frac{d \theta}{d t}=-\frac{(v+2)}{2 a_{\varphi} \omega\left(a_{\varphi}\right)} \varepsilon c a(v+1,1, \bar{\theta}) \bar{f}\left(a_{\varphi}, \dot{\bar{\theta}}\right) .
\end{aligned}
$$

The obtained system of differential equation can be much more simplified due to the following reasoning: amount of oscillations amplitude and frequency change at one period is small, as maximum value of resistance forces is much smaller than maximum value of suspensions restoring force. This is the reason for phase averaging [18] of the equation right parts:

$$
\frac{d a}{d t}=-\frac{\left(\alpha_{1} a^{s+2}+\alpha_{2} b^{s+2}\right) a}{2 \Pi I_{0}}\left(\frac{2 a \omega(a)}{(v+2)}\right)^{s-1} \Gamma\left(\frac{1}{v+2}\right) \Gamma\left(\frac{s+2}{2}\right) \Gamma^{-1}\left(\frac{1}{v+2}+\frac{s+2}{2}\right),
$$


$\dot{\vartheta}=\frac{(v+1) \Delta_{\infty \dot{\alpha}}\left(c_{1} a^{v+1}-c_{2} b^{v+1}\right)}{2 \Pi I_{0}} \Gamma\left(\frac{1}{v+2}\right) \Gamma\left(\frac{v+2}{2}\right) \Gamma^{-1}\left(\frac{1}{v+2}+\frac{v+2}{2}\right)$,

where $\Pi-$ half-period of used periodic Ateb - functions, i.e. $\Pi=\sqrt{\pi} \Gamma(1 /(v+2)) \Gamma^{-1}(1 / 2+1 /(v+2))$.

Then, at determining dynamic values of WV pressure force on bearing surface we restrict to the extreme values of $\mathrm{CP}$ inertia forces moment, i.e.

$\bar{M}_{A}^{\circ}= \pm I_{A} \omega^{2}\left(a_{\varphi}\right)= \pm \frac{I_{A}(v+2)}{2 I_{0}} \cdot\left(c_{1} a^{v+2}+c_{2} b^{v+2}\right) \cdot a_{\varphi}^{v+1}$

where $I_{A}-\mathrm{CP}$ inertia moment of about contact point of steered wheel and a road. It's, by Huygens-Steiner theorem [7], equal to $I_{A}=I_{o}+P / g\left(a^{2}+c^{2} / 4\right)=\frac{P}{3 g}\left(4 a^{2}+b^{2}+c^{2}\right)$. It makes possible to find minimum value of steered wheels pressure force on bearing surface

$G_{k}=N_{1}=P+P_{1}-\frac{P a}{a+b}+ \pm \frac{2(v+2)}{(a+b)} \frac{\left(4 a^{2}+b^{2}+c^{2}\right)}{\left(4 a^{2}+4 b^{2}+c^{2}\right)}\left(c_{1} a^{v+2}+c_{2} b^{v+2}\right) \cdot a_{\varphi}^{v+1}$,

and then tire angular rigidity - vertical axis ratio $-c_{\omega}=k G_{k}$. Coefficient $k$ is determined by empirical dependence $k=(9 \ldots 11) \cdot 10^{-3} \mathrm{Hm} /$ degr. Thus, dynamic resistance moment of turning tire $M_{\varphi}$ taking into account $\mathrm{CP}$ oscillations takes the following value

$M_{\varphi}=k\left[P+P_{1}-\frac{P a}{a+b}+ \pm \frac{2(v+2)}{(a+b)} \frac{\left(4 a^{2}+b^{2}+c^{2}\right)}{\left(4 a^{2}+4 b^{2}+c^{2}\right)}\left(c_{1} a^{v+2}+c_{2} b^{v+2}\right) \cdot a_{\varphi}^{v+1}\right] \Theta$,

where $\Theta$ - turning angle of steered wheel against pin axle (axle against which the wheel is turning).

\section{RESULTS AND DISCUSSION}

We assume, that dynamic resistance moment of turning tire is proportional to tire turning angle. The above mentioned research allows to write the WV steerability condition (taking into account CP oscillations) $M_{\varphi} \leq M_{\varphi \max }$, where $M_{\varphi \max }$ - critical value on tire road capacity of resistance moment of turning tire, occurring at complete sliding of tire components, which contact with the bearing surface [1]. As a critical value resistance moment of turning tire we may assume the maximum value of static resistance moment of turning tire: $M_{\varphi \max }=k\left(P+P_{1}+P b /(a+b)\right) \Theta_{\max }, \Theta_{\max }-$ critical value of steered wheel turning angle, that can be found empirically for different types of covering. In this case to determine limiting angle of steered wheel turning taking into account longitudinal oscillations of CP we obtain the dependence;

$$
\left[P+P_{1}-\frac{P a}{a+b}+ \pm \frac{2(v+2)}{(a+b)} \frac{\left(4 a^{2}+b^{2}+c^{2}\right)}{\left(4 a^{2}+4 b^{2}+c^{2}\right)}\left(c_{1} a^{v+2}+c_{2} b^{v+2}\right) \cdot a_{\varphi}^{v+1}\right] \Theta \leq\left(P+P_{1}-\frac{P a}{a+b}\right) \Theta_{\text {max }}
$$


From obtained ratio we find the value of critical dynamic angle of turning $\bar{\Theta}$ depending on longitudinal-angular oscillations amplitude of $\mathrm{CP}$ and parameters describing $\mathrm{CP}$ force characteristic

$$
\bar{\Theta}=\frac{\left(P+P_{1}\right)(a+b)-P a}{\left(P+P_{1}\right)(a+b)-P a+ \pm 2(v+2) \frac{\left(4 a^{2}+b^{2}+c^{2}\right)}{\left(4 a^{2}+4 b^{2}+c^{2}\right)}\left(c_{1} a^{v+2}+c_{2} b^{v+2}\right) a_{\varphi}^{v+1}} \Theta_{\max }
$$

Having replaced in obtained ratio rigidity coefficients for $\mathrm{CP}$ static deformation, dependence of dynamic angle of turning critical value takes the following form;

$$
\bar{\Theta}=\frac{\left.\left(P+P_{1}\right)(a+b)-P a\right)(1+\kappa) \Delta_{\mathrm{st}}^{v+1}}{\left.\left(P+P_{1}\right)(a+b)-P a\right)(1+\kappa) \Delta_{\mathrm{st}}^{v+1}+ \pm 2 P(v+2)\left(a^{v+2}+\kappa b^{b+2}\right)\left(4 a^{2}+b^{2}+c^{2}\right) a_{\varphi}^{v+1} /\left(4 a^{2}+4 b^{2}+c^{2}\right)} \Theta_{\text {max }}
$$

As we expected, when $a_{\varphi}=0$ from dependence Eq. (19) we obtain the known result: $\Theta_{\max }=\bar{\Theta}$. So, on Figure 3 the dependence of ratio critical value of turning dynamic angle - static value of the same angle $(\eta)$ on longitudinal-angular oscillations amplitude at different values of force characteristics of WV non-linear suspension (parameters $v$ and $\left.\Delta_{\text {st. }}\right)$ at the following values of other parameters $P=10^{5} \mathrm{~N}, P_{1}=10^{4} \mathrm{~N}, a=c=1 \mathrm{~m}$, $b=1,1 m, \kappa=1,2$.

The results deal with critical dynamic angle of turning during car motion along rectilinear road intervals. In case of curvilinear road intervals to calculate the critical value of driving speed one must additionally take into account forces of inertia of some its parts [22], transverse force of friction of wheels and road (including the force component dependence on the displacement angle of wheels) [23-26].

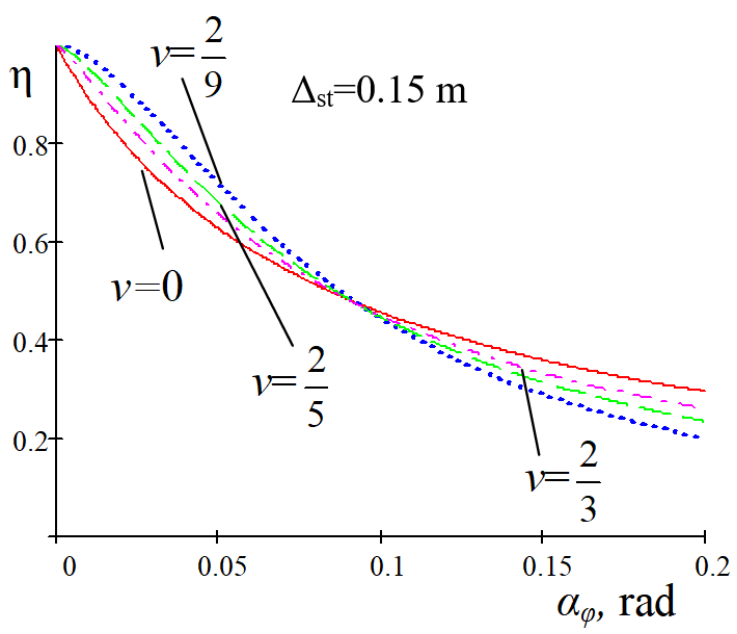

(a)

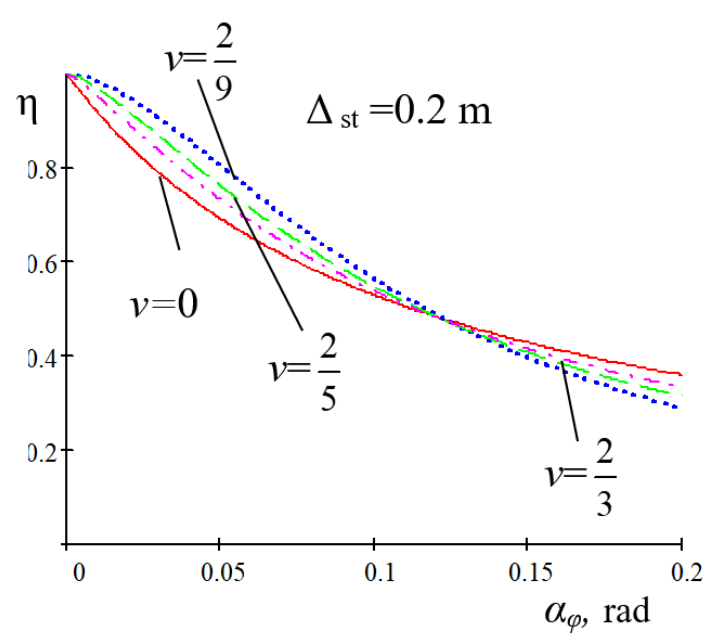

(b) 


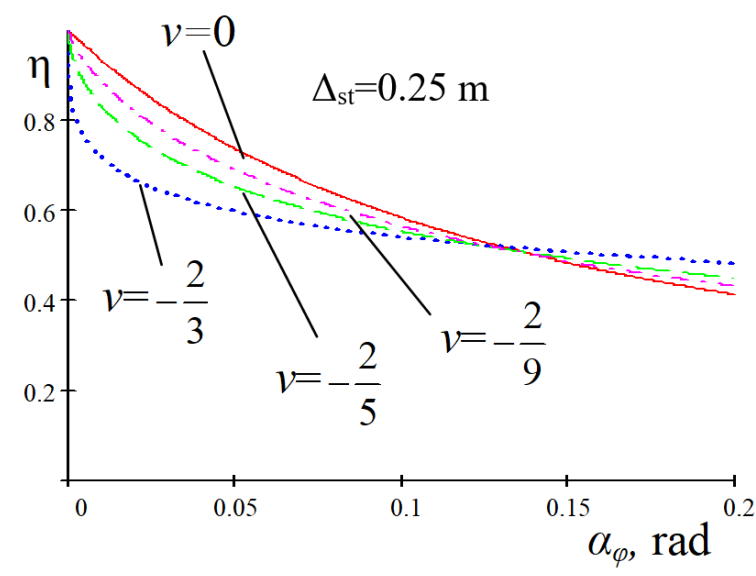

(c)

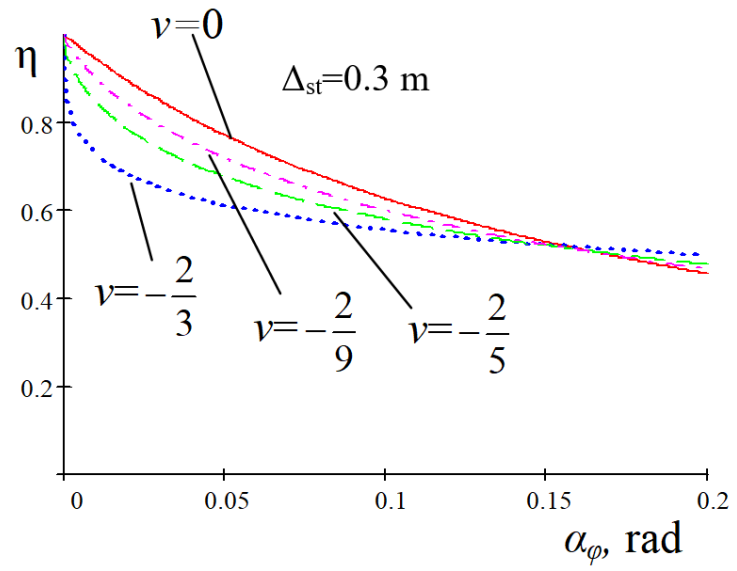

(d)

Figure 3. The dependence of ratio critical value of turning dynamic angle - static value of the same angle on longitudinal angular oscillations amplitude at different values of force characteristics of WV non-linear suspension.

As the research of car steerability is the most important for high speed of its motion on curvilinear road intervals and so the angles of turning of steered wheels are small, we may assume with sufficient degree of adequacy:

a) Transverse component force acting on the wheel and caused by its displacement $F$ as a function of displacement angle is described by linear dependence $F(U)=f N U$ (for certain type of road pavement its value of coefficient $f$ corresponds to the value of rate of change to the correspondent curve. Thus, $F_{1}(U)=f N_{1} U_{1}$ and $F_{2}(U)=f N_{2} U_{2}$ - are respectively the values of transverse component of friction force caused by front and rear wheels displacement, and $U_{1}$ and $U_{2}$ are the values of displacement angles for the wheels of front and back axes;

b) Transverse component of friction force caused by its interaction with seating is proportional to its pressure force on the latter with proportion factor $f_{1}$ and is found for the front and rear wheels by dependencies [1] $\bar{F}_{1}=f_{1} N_{1}$ and $\bar{F}_{2}=f_{1} N_{2}$;

c) Centrifugal inertia forces of its spring and non-spring weight are perpendicular to the car longitudinal axis and their resultant line of actions $-\Phi^{\mathrm{iH}}$ passes across the car center of mass. Values of centrifugal inertia force are found by dependence $\Phi^{\mathrm{iH}}=\frac{\mathrm{P}+\mathrm{P}_{1}+\mathrm{P}_{2}}{\mathrm{~g}} \frac{\mathrm{V}^{2}}{\rho}$, where $V-$ driving speed and $\rho$ turning radius. Its components on front and rear are equal respectively $\Phi_{1}^{\mathrm{iH}}=\frac{\left(\mathrm{P}+\mathrm{P}_{1}+\mathrm{P}_{2}\right) \mathrm{a}}{(\mathrm{a}+\mathrm{b})} \frac{\mathrm{V}^{2}}{\rho}$, $\Phi_{2}^{\mathrm{iH}}=\frac{\left(\mathrm{P}+\mathrm{P}_{1}+\mathrm{P}_{2}\right) \mathrm{b}}{(\mathrm{a}+\mathrm{b})} \frac{\mathrm{V}^{2}}{\rho}$.

Thus, the above given information allows to obtain the following dependencies to estimate the steerability of front and rear wheels. 


$$
\begin{aligned}
& \frac{\left(\mathrm{P}+\mathrm{P}_{1}+P_{2}\right) a}{(a+b)} \frac{V^{2}}{\rho}=N_{1}\left(f_{1}+f U_{1}\right), \\
& \frac{\left(\mathrm{P}+\mathrm{P}_{1}+P_{2}\right) b}{(a+b)} \frac{V^{2}}{\rho}=N_{2}\left(f_{1}+f U_{2}\right)
\end{aligned}
$$

The given dependencies determine taking into account ratio Eq. (5), critical values of speed considering front and rear wheels steerability and taking into account angles of displacement and vibrations of sprung part:

$$
\begin{aligned}
& V_{1}=\left\{\frac{\left(f_{1}+f U_{1}\right) \rho(a+b)}{\left(\mathrm{P}+\mathrm{P}_{1}+P_{2}\right) a}\left[P \frac{b}{a+b}+P_{1}-\frac{2 P(v+2) a\left(4 a^{2}+b^{2}+c^{2}\right)}{(a+b)^{2}\left(4 a^{2}+4 b^{2}+c^{2}\right)}\left(c_{1} a^{v+2}+c_{2} b^{v+2}\right) a_{\phi}^{v+1}\right]\right\}^{\frac{1}{2}}, \\
& V_{2}=\left\{\frac{\left(f_{1}+f U_{2}\right) \rho(a+b)}{\left(\mathrm{P}+\mathrm{P}_{1}+P_{2}\right) b}\left[P \frac{a}{a+b}+P_{2}-P \frac{2(v+2) a\left(4 a^{2}+b^{2}+c^{2}\right)}{(a+b)^{2}\left(4 a^{2}+4 b^{2}+c^{2}\right)}\left(c_{1} a^{v+2}+c_{2} b^{v+2}\right) a_{\phi}^{v+1}\right]\right\}^{\frac{1}{2}},
\end{aligned}
$$

Hence, all this obtained material allows to find the critical value of speed taking into account the car steerability as $\bar{V}=\min \left\{V_{1}, V_{2}\right\}$.

Most leading automobile makers are doing intense research in this field. Among them the following car manufacturers: Mercedes Benz, General Motors, Ford, Opel, Audi, Renault, Citroen, Volvo, Mitsubishi, Toyota, Nissan. The market trend has changed: when in 1980s active suspensions were used only in racing cars of Formula 1, in 1990s - in expensive off-road vehicles and SUV, in 2000 - in expensive passenger cars, at present almost all car makers are speaking about active suspensions implementation [27]. The special attention to active suspensions models is paid by developers of specialized vehicles - reanimobiles, vehicles for special cargo transportation [28].

Mathematical model use allowed to determine analytically the influence of various factors (structural parameters) on ride quality and exact enough description of car vibration at motion on specific pavement. The described method of study the influence of longitudinal-and-angular oscillations of wheeled vehicles with non-linear elastic characteristics of suspension system on their steerability has been implemented in the papers [29-31].

\section{CONCLUSION}

The obtained theoretical results and constructed on their basis graphs of dependencies have shown:

a) Longitudinal-angular oscillations of WV cushion part considerably reduce the critical value of dynamic angle of turning. Moreover, its value is smaller for larger amplitudes of oscillations;

b) Critical value of dynamic turning angle for cushion system with regressive law of dampers elastic characteristics change at small amplitudes of longitudinal-angular oscillations is smaller than for suspensions with linear law of its change and vice versa - it is larger for large amplitudes of longitudinal-angular oscillations. For the cushion system with progressive law of restoring force change the inverse 
relation takes place. When used in wheeled vehicles, the cushioning system with progressive characteristics of dampers restoring force at $2 / 9<v<2 / 3$ increases the critical value of steering angle up to $18 \%$ in comparison with its linear analog at amplitude of lateral-angular oscillations $0.05 \mathrm{rad}$ and dampers static deformation $0.2 \mathrm{~m}$, and for large amplitudes of lateral-angular oscillations - the use of regressive characteristics of cushioning system $-2 / 3<v<-2 / 9$ increases the critical value of steering angle (up to $10 \%$ ) in comparison with a linear analog;

c) For more rigid suspensions (bigger value of parameter $v$ and smaller value of static deformation) the critical value of dynamic turning angle is smaller for small amplitudes of longitudinal-angular oscillations and bigger for big amplitudes of longitudinal-angular oscillations. Namely, the rise of static deformation of cushion part at $v=2 / 5$ and amplitude of lateral-angular oscillations $0.04 \mathrm{rad}$ increases the value of steering angle up to $14.7 \%$ in comparison with cushion system with linear characteristics of restoring force. At the same time the obtained results may serve as a basis for the development of program product of adaptive (steerable) cushion system. Such investigations for the suspension type under consideration have been conducted by the authors and they will be the subjectmatter for further publications.

\section{REFERENCES}

[1] Soltus AP. Theory of car performance characteristics. Kiev: Aristei; 2010.

[2] Soliman A, Moustafa, S, and Shogae, A. Parameters affecting vehicle ride comfort using half vehicle model. SAE Technical Paper. 2008; 2008-01-1146.

[3] Deb A, Joshi D. A study on ride comfort assessment of multiple occupants using lumped parameter analysis. SAE Technical Paper. 2012; 2012-01-0053.

[4] Zandieh A. Dynamics of a three - wheel vehicle with tadpole design. A thesis presented to the University of Waterloo in fulfillment of the thesis requirement for the degree of Master of Science in Mechanical Engineering, Ontario, Canada; 2014. Retrieved from https://uwspace.uwaterloo.ca/bitstream/,

[5] Previati G, Gobbi M, Mastinu G. Friction coefficient on snowy and icy surfaces of pneumatic tires fitted with or without anti-skid devices. SAE Technical Paper. 2006; 2006-01-0560.

[6] Grubel MH, Krasyuk OP, Nanivskyy RA, Sokil MB. Vertical oscillations of wheeled vehicles cushion part under random disturbance conditions, Scientific notes of Lutsk National Technical University. 2014; 46:112-116.

[7] Kuzio IV, Sokil BI, Palyukh VM. Suspension parameters effect on wheeled vehicles non-linear oscillations Bulletin of National University Lviv Polytechnic. Dynamics, durability and design of machines and instruments. 2007; 588:49-52.

[8] Bogdevičius M, Junevičius R. Dynamic processes of the retrofited rear suspension of the vehicle. Transport, 2004; 19: 262-268.

[9] Pečeliūnas R, Lukoševičienè O, Prentkovskis OA mathematical model of the vibrating system equivalent to the vehicle in the mode of emergency braking. Transport. 2003; 18: 136-142.

[10] Tamboli JA, Joshi SG. Optimum design of a passive suspension system of a vehicle subjected to actual road random excitations. J Sound Vib. 1999; 219: 193205. 
[11] Taghavifar H, Mardani A, Off-road Vehicle Dynamics: Analysis, Modelling and Optimization. Springer: Mechanical Engineering, Switzerland; 2016.

[12] Gillespie TD. Fundamentals of Vehicle Dynamics. Society of Automotive Engineers: Warrendale, PA.; 1992.

[13] Rill G. Road Vehicle Dynamics: Fundamentals and Modeling. CRC Press, Boca Raton FL; 2012.

[14] Balthazar M, Mook DT, Weber HI, Brasil RMLRF, Fenili A, Belato D, Felix JLP. An overview on non-ideal vibrations. Meccanica. 2003; 38: 613-621.

[15] Blekhman, Vibrational Mechanics: Nonlinear Dynamic Effects, General Approach, Applications. World Scientific, Singapore; 2000.

[16] Grubel MH, Nanivskyy RA, Sokil MB. Effect of elastic suspension restoring force on wheeled vehicles stable motion, Prospects for SV armament and military engineering development: Abstracts of International Scientific and Technical Conference, Lviv: ACB; 2014.

[17] Cole J. Disturbance methods in applied mathematics. Moscow: Mir; 1972.

[18] Boholyubov NN, Mytropolskyy YA. Asymptomatic methods in non-linear oscillations theory. Moscow: Science: 1974.

[19] Senyk PM. Incomplete Beta-function inversion. Ukrainian Mathematical Journal. 1969; 21: 325-333.

[20] Senyk PM, Sokil BI. About U-method use for one class of oscillation system. Report Academy of Sciences USSR. 1977; 1: 12-16.

[21] Sokil BI, Senyk PM. Parameters determination of non-linear oscillation system on amplitude - frequency characteristic. Mathematical methods and physical and mechanical fields. 1977; 7: 94-99.

[22] Volkov VP, Vilskyi HB. Theory of car motion. Sumy: University Book; 2010.

[23] Savkoor AR. Boundary Conditions on Models for Predicting Tyre to Road Traction. Vehicle System Dynamics, 1992; 21: 178-185.

[24] Gim G, Nikravesh PE. An analytical model of pneumatic tires for vehicle dynamic simulations. 3. Validation against experimental-data. International Journal of Vehicle Design. 1991; 12(2): 217-28.

[25] Lyashuk O, Pyndus T, Marunych O, Sokil M. Longitudinal-angular oscillation of wheeled vehicles with non-linear power characteristics of absorber system. Scientific Journal of the Ternopil National Technical University. 2016; 83: 82-89.

[26] Lyashuk O, Sokil M, Vovk Y, Tson O, Dzyura V. The impact of the kinematic parameters of bounce and pitch motions of sprung mass on wheeled vehicles handling. Scientific Journal of Silesian University of Technology. Series Transport. 2017; 97: 81-91.

[27] Pavlenko VM, Kryvoruchko OO. Modern state of development of active suspensions of motor cars. Bulletin NTU KhPI, Automobile and tractor manufacture. 2014; 1052: 54-60.

[28] Serta E, Boyraz P. Optimization of suspension system and sensitivity analysis for improvement of stability in a midsize heavy vehicle. Engineering Science and Technology, an International Journal. 2017; 20: 997-1012.

[29] Artyushenko A, Suyarkov O. Study of influence of suspension characteristics of small size car on ride quality and its modernization. Bulletin NTU KhPI. Series: Transport mechanical engineering. 2013; 1004: 21-27.

[30] Podryhalo MA, Korbko MI, Klets DM. Evaluation of automobile dynamic ability. Automobile and tractor manufacture: Bulletin NTU KhPI. 2008: 134-137. 
[31] Podryhalo MA, Volkov VP, Boboshko AA, Pavlenko VA, Baitsur MV, Nazarov AI, Aleksev VO. Wheeled vehicles resistance to skidding whilst breaking and ways of its rising. Kharkiv: KNARU; 2006. 